\title{
Ankle Foot Orthosis Improves Functional Ambulation and Balance in Patients with Peripheral Nerve Palsy
}

Tokio Kinoshita ${ }^{1}$, Yukihide Nishimura ${ }^{1 *}$, Takeshi Nakamura², Daisuke Kojima1, Yuta Sakurai ${ }^{1}$, Yoshinori Yasuoka1, Yoshi-ichiro Kamijo', Hiroyoshi Fujiwara $^{3}$, Toshikazu Kubo ${ }^{3}$, Tadashi Sumiya ${ }^{1}$ and Fumihiro Tajima ${ }^{1}$

${ }^{1}$ Department of Rehabilitation Medicine, Wakayama Medical University, 811-1 kimiidera, Wakayama city, Wakayama 641-8509, Japan

${ }^{2}$ Department of Rehabilitation Medicine, School of Medicine, Yokohama City University, 3-9 Fukuura, Kanazawak-ku, Yokohama, Kanagawa 236-0004, Japan

${ }^{3}$ Department of Orthopaedics, Graduate School of Medical Science, Kyoto Prefectural University of Medicine, Yoshida-Konoe-cho, Sakyo-ku, Kyoto 606-8501, Japan

\begin{abstract}
Background: Several studies have examined the benefits of ankle foot orthosis (AFO) in patients with hemiparesis, but little is known about their effects in patients with peripheral nerve palsy. The purpose of this study was to compare the effects of AFO on functional ambulation in patients with hemiplegia and unilateral lower-extremity palsy.

Subjects and methods: This study design was Case control study in rehabilitation units of five hospitals in Japan. The study subjects were 50 normal subjects (control), 49 patients with post-stroke hemiparesis (PSH), and 13 with lower extremity palsy (LEP) due to peripheral nerve palsy. Functional ambulation was assessed with AFO and without AFO by 10 -meter maximum walking speed (10 MMWS) and the distance walked in a 6-minute walking test (6 MWT). Functional balance was assessed using the Berg balance scale.
\end{abstract}

Results: The 10 MMWS test showed significantly lower walking speed in PSH and LEP without AFO compared with the control group, and that the speed was faster in the two patient groups with AFO than without AFO. The distance walked during the 6 MWT was significantly shorter in PSH and LEP than the control, but it increased significantly in both groups after wearing the AFO. Furthermore, the significant difference between the control and LEP groups disappeared after wearing the AFO. The Berg balance scale was significantly lower in PSH and LEP groups compared with the control group, but improved significantly with AFO.

Conclusion: The results suggest that AFO is beneficial for patients with PSH and those with LEP through improvement of walking distance, walking speed, and functional balance. Many physicians and technicians often avoid the use AFO. However, AFO improved not only walking ability but also functional balance in both groups of patients. Physicians and technicians are encouraged to consider the use of AFO in LEP.

Keywords: Ankle foot orthosis; Hemiparesis; Peripheral nerve palsy; Walking distance; Walking speed; Functional balance

Abbreviations: AFO: Ankle Foot Orthosis; BBS: Berg Balance Scale; Control: Healthy Individuals; PSH: Post-Stroke Hemiparesis; LEP: Lower Extremity Palsy; mRs: modified Rankin scale; NIHSS: National Institute of Health Stroke Scale; 6 MWT: 6-Minute Walking Test; 10 MMW: 10-Meter Maximum Walking Speed

\section{Introduction}

In Japan, there are estimated 1,235,000 individuals with stroke who receive continuous medical care in hospitals [1]. According to the 2013 report by the Ministry of Health, Labour and Welfare, stroke is ranked the fourth cause of death following cancer, cardiac disease, and pneumonia [2]. However, while stroke was associated with high mortality in the past, today, many patients survive after stroke though they suffer from various forms of disabilities.

The main functional outcome of human ambulation includes safe walking and efficient movement from one place to another [3]. Normal locomotion involves selective muscle control and normal range of motion of multitude of joints. On the other hand, abnormal motor control and limb deformities are common complications in patients with stroke. The mobility of many stroke survivors is limited [4], and most identify walking as a top priority in any rehabilitation program [5]. Impaired walking in stroke is due to various factors such as muscle weakness, spasticity, and discoordination of lower limb muscle activity [6]. In addition to impeding endurance, gait impairment limits walking distance and velocity, which reduces quality of life of stroke survivors. Slow walking velocity is associated with limitation in home and community activities, increased risk of falls [7], and increased mortality [8]. Therefore, it is imperative for physical therapists to identify appropriate strategies and interventions to optimize walking in this population.
Lower motor neuron lesions are associated with damage to nerve fibers traveling from the anterior horn of the spinal cord to the relevant muscles, such as that seen in cauda equine disorder, radiculopathy and common peroneal nerve palsy. Cauda equina is caused by external pressure on nerves and manifests clinically by bladder/rectal disorders, sensory and motor deficits of the lower limbs. The main causes of cauda equina are lumbar spinal canal stenosis, herniated disk, tumor and abscess, trauma and injury following spinal anesthesia or local inflammation. In lumbar radiculopathy, the spinal nerve roots are irritated or compressed by certain pathologies, such as lumbar disk herniation, spinal stenosis, osteophyte formation, spondylolithesis, foraminal stenosis, or other degenerative disorders. Peroneal nerve palsy results from pressure by the fibula head in patients on protracted bed rest or those with plaster cast fixation, space-occupying lesions (e.g., ganglion, tumor, hematoma), bone fracture or trauma. There is little information on the incidence of lumbar spinal canal stenosis, lumbar herniated disk, and common peroneal nerve paralysis in Japan.

*Corresponding author: Yukihide Nishimura, MD, PhD, Department of Rehabilitation Medicine, Wakayama Medical University, 811-1 Kimiidera, Wakayama city, Wakayama 641-8509, Japan, Tel: +81 (73) 441-0664; Fax: +81 (73) 446-6475; E-mail: yuki-n@wakayama-med.ac.jp

Received October 13, 2016; Accepted October 17, 2016; Published October 19 2016

Citation: Kinoshita T, Nishimura Y, Nakamura T, Kojima D, Sakurai Y, et al. (2016) Ankle Foot Orthosis Improves Functional Ambulation and Balance in Patients with Peripheral Nerve Palsy. J Spine 5: 337. doi: 10.4172/2165-7939.1000337

Copyright: (c) 2016 Kinoshita T, et al. This is an open-access article distributed under the terms of the Creative Commons Attribution License, which permits unrestricted use, distribution, and reproduction in any medium, provided the original author and source are credited. 
While patients with stroke and lower motor neuron disorders have walking disorders, the two groups differ in other clinical features. For example, patients with lower motor neuron lesions have flaccid muscle paralysis and sensory paralysis, compared with spastic paralysis and various neurological deficits and disabilities in stroke patients, such as paralysis of the arm, leg, and trunk, communication disorder, cognitive impairment and visual-spatial perception disorders.

Orthosis is defined by the International Standards Organization as "an externally applied device used to modify the structural and functional characteristics of the neuromuscular and skeletal system" [9]. The ankle foot orthosis (AFO) device usually encompasses the ankle joint and the whole or part of the foot. AFO are intended to control motion, correct deformity and/or compensate for weakness (Figure 1). AFO can be designed with sufficient mechanical lever arms for direct control of the ankle complex with indirect effect on the knee joint. There are many commercially different types of AFO, which may vary in their biomechanical design (including desired mechanical force systems, any joint or articulation, alignment and range of motion), materials and components. AFO can be rationally prescribed based on their biomechanical function. The design of the AFO should be considered and best practice points have been recommended for different AFO designs used in the management of different conditions.

AFO is used in the management of patients with walking difficulties. AFO serves to provide foot and ankle stabilization during weightbearing and lifting the toes while stepping [10]. AFO can be useful in accommodating the limitations in mobility associated with stroke [11]. The use of AFO is, however, controversial. Some physiatrists and physiotherapists discourage the use of orthosis $[12,13]$, arguing that it can prevent or delay recovery of normal movement. One published review concluded that AFO had little effect on muscle activity in the paretic leg, although its use resulted in immediate kinematic and temporal improvement [10]. Another more recent review concluded that AFO improves walking function [14].

AFOs are commonly used in patients with peripheral palsy, and are usually prescribed for patients with degree of paresis of less than 3 on the manual tibialis anterior or peroneus muscle test. However, little is known about the biomechanical and functional effects of AFO on functional ambulation in patients with lower motor neuron lesions, although these issues have been examined in stroke patients $[15,16]$. The purpose of this retrospective study was to compare the effects of AFO on functional ambulation in individuals with hemiplegia secondary to stroke and unilateral lower-extremity palsy using quantifiable outcome measures.

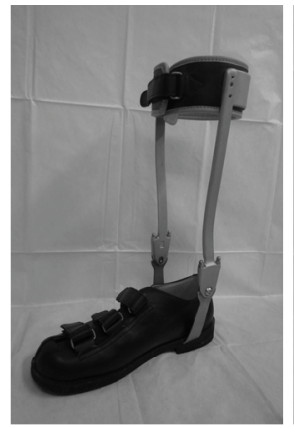

A)

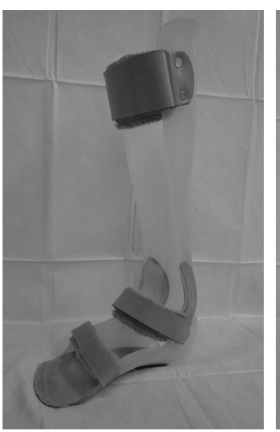

B)

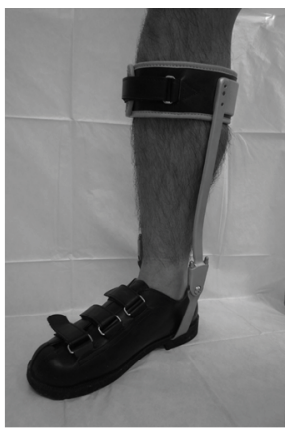

Figure 1: Types of ankle foot orthosis (AFO). [Type A: articulated metal AFO with double bars attached to the outsole of a normal shoe. Type B: solid polypropylene AFO]

\section{Methods}

\section{Participants}

The study subjects were 50 healthy individuals (control), 49 patients with post-stroke hemiparesis (PSH), and 13 patients with lowerextremity palsy (LEP) due to peripheral nerve palsy (cauda equina disorder $\mathrm{n}=4$, radiculopathy $\mathrm{n}=4$, sciatic nerve palsy $\mathrm{n}=1$, common peroneal nerve palsy $n=4$ ). The degree of paresis in patients with LEP was less than 3 in manual tibialis anterior and peroneus muscle test. AFO had been prescribed for PSH and LEP patients for the management of motor dysfunction, functional ambulation and sensory disturbance. The time of prescription of AFO and time since the use of AFO varied from one subject to another. The inclusion criteria were the followings: 1) no history of injury or pathology in the uninvolved lower limb;2) ability to walk independently or with supervision for 6 minutes, both with and without AFO; and 3) wearing AFO for more than $50 \%$ of the walking time. Individuals with significant orthopedic, neuromuscular, or neurological pathologies or history that would interfere with walking or limit the range of motion of the legs were excluded from the study. The medical records of the PSH and LEP patients were scanned for various clinical and laboratory data and these, together with data of the normal subjects, are presented in Table 1. For LEP subjects, the onset of peripheral nerve palsy was determined by the attending physician. There were no significant differences in age and height between the three groups. Subjects of the LEP group were significantly heavier than the control, and the body mass index was significantly greater in the LEP group than the PSH and control groups. The modified Rankin scale (mRs) was $2.8 \pm 0.7$ in the PSH and $2.3 \pm 0.5$ in the LEP patients [17].

The study protocol was approved by the ethics review committee of Wakayama Medical University and conformed to the Declaration of Helsinki. A signed informed consent was obtained from each subject after a thorough explanation of the purpose and risks of the study.

\section{Study protocol and measurements}

For the purpose of this study, all subjects were seen at the Outpatient Clinic. All participants received detailed instructions on each test. Mobility was evaluated by skilled physiotherapists and physiatrists. All tests were performed in a single day in each subject. The order of testing with and without AFO was randomized in a cross-over design. Members of the study team provided supervision and noncontact guard during all trials for safety.

\begin{tabular}{|c|c|c|c|}
\hline Variable & Control $(\mathbf{n = 5 0}$ & PSH $(\mathbf{n = 4 9 )}$ & LEP $(\mathbf{n = 1 3})$ \\
\hline Age (years) & $66.0 \pm 14.0$ & $62.3 \pm 11.0$ & $54.4 \pm 19.2$ \\
\hline Gender (female/male) & $23 / 27$ & $11 / 38$ & $5 / 8$ \\
\hline Height (cm) & $159.5 \pm 9.2$ & $163.0 \pm 9.2$ & $163.6 \pm 9.7$ \\
\hline Weight (kg) & $58.9 \pm 11.1$ & $61.7 \pm 10.6$ & $72.2 \pm 15.7^{*}$ \\
\hline Body mass index (kg/m²) & $23.1 \pm 3.8$ & $23.2 \pm 3.8$ & $26.9 \pm 4.7^{*}, \#$ \\
\hline Time since onset (months) & - & $48.6 \pm 42.6$ & $15.5 \pm 14.0$ \\
\hline NIHSS score (0-42) & - & $4.0 \pm 2.6$ & - \\
\hline Modified Rankin scale (0-6) & - & $2.8 \pm 0.7$ & $2.3 \pm 0.5$ \\
\hline Assistive device (n, \%) & & & \\
\hline None & $50(100)$ & $7(14.3)$ & $7(54)$ \\
\hline Single point cane & 0 & $38(77.6)$ & $6(46)$ \\
\hline Quad cane & 0 & $4(8.1)$ & 0 \\
\hline Ashworth scale $(0-5)$ & - & $1.8 \pm 1.30$ & - \\
\hline
\end{tabular}

Data are mean $\pm S D$. ${ }^{*} p<0.05$, compared with the control group. ${ }^{*} p<0.05$, compared with the PSH and LEP groups. AFO: ankle foot orthosis, BBS: Berg Balance Scale, PSH: post-stroke hemiparesis,

NIHSS: National Institute of Health Stroke Scale, LEP: lower extremity palsy

Table 1: Clinical characteristics of the study participants. 
Gait velocity: Each subject was asked to walk 16-meter straight course at own maximum walking speed. Gait velocity was calculated from the time registered within the center of 10 meters. Walking speed was recorded using a stopwatch (model SSBJ018, Seiko, Tokyo, Japan) [18]. The test was repeated twice, and data of the fastest walk were adopted.

Walking endurance: Walking endurance was assessed in terms of the distance walked in the 6 minutes walking test (6 MWT) [19]. The subjects walked up and down around 30 meters' corridor using their usual walking aids (i.e., cane). Verbal encouragement was provided at 1 , 3 , and 5 minutes during the walk, including: "You're doing a good job" (minute 1), "You're halfway done" (minute 3), and "You have 1 minute to go" (minute 5). To ensure safety, the investigator walked slightly behind but not beside the subjects, so as to avoid influencing their self-selected walking pace. Subjects were allowed to stop and rest as they deemed necessary. The 6 MWT distance was recorded using a measuring wheel (Land Art; HSM-101. Kagoshima, Japan). The distance covered, to the nearest centimetre, was recorded.

Berg balance scale: The Berg Balance Scale (BBS) is a clinical measure of functional balance and assesses 14 different tasks (sitting with arms folded, rising from sitting position, standing, standing with eyes closed, standing with feet together, standing to sitting, transfer from one surface to another, reaching forward in standing, picking up objects from the floor, turning around in a full circle, turning to look behind, standing with one foot in front, placing alternate foot on stool and standing on one leg) on a scale of 0 to 4 , with 0 representing inability to complete the entire task. The total score ranges from 0 to 56. Scoring is based on the ability to meet specific time and distance goals in each task. The test was designed for elderly and rehabilitation patients; but has been validated in early post-stroke patients and has very high intra- and interrater reliability [20].

National Institute of Health stroke scale: The National Institute of Health Stroke Scale (NIHSS) is a 15-item neurologic examination stroke scale used to evaluate the effect of acute cerebral infarction on the levels of consciousness, language, neglect, visual-field loss, extraocular movement, motor strength, ataxia, dysarthria, and sensory loss. The score of NIHSS ranges from 0 to 42 points, with 0 indicating normal neurological function and 42 indicating no function [21].

Modified Rankin Scale score: The modified Rankin scale defines six levels of disability and 1 for death: $0=$ no symptom at all; $1=$ no significant disability despite symptoms, ability to carry out all usual duties and activities; 2 = slight disability, inability carrying out all previous activities but ability to look after own affairs without assistance; 3 = moderate disability, requiring some help but ability to walk without assistance; $4=$ moderately severe disability, inability to walk without assistance and inability to attend to own bodily needs without assistance; 5 = severe disability, bedridden, incontinent, and requiring constant nursing care and attention; and $6=$ dead. Individual scores in the mRS describe clinically distinct functional state of the patient [17]
Ashworth scale: The Ashworth Scale measures spasticity in patients with central nervous system lesions or neurological disorders. The Ashworth Scale is a quick and easy measure that can assist a clinician's assessment of spasticity during passive soft-tissue stretching. The test assesses resistance to passive movement of a joint with varying degrees of velocity. Scores range from $0-4$, with score 0 indicates no resistance, 1 mild resistance, score 2 between 1 and 3 , score 3 indicates difficult to passive motion, and score 4 indicates rigidity [22].

\section{Statistical analysis}

All data were expressed as mean $\pm \mathrm{SD}$. The percent change in each parameter represented the mean value measured with the patient wearing the AFO divided by the value measured without AFO. Demographic data and all outcome measures were analyzed by descriptive statistics. All data depicted in Tables 1 and 2 were analyzed by Kruskal-Wallis test. We also used the Dunn's test for subsequent post-hoc test to determine significance of differences among the three groups (PSH, LEP, and the control). The Mann-Whitney U test was used for differences between the PSH and LEP groups, and also with AFO and without AFO. A $P$ value less than 0.05 was considered statistically significant. All statistical analyses were conducted using the Graph Pad Prism 6 software (Graph Pad, La Jolla, CA).

\section{Results}

\section{The 10-meter maximum walking speed test}

The 10-meter maximum walking speed was significantly slower in the PSH $(0.50 \pm 0.41 \mathrm{~m} / \mathrm{sec})$ and LEP $(1.11 \pm 0.31 \mathrm{~m} / \mathrm{sec})$ groups without AFO compared with the control group $(1.88 \pm 0.45 \mathrm{~m} / \mathrm{sec})$. Also, the PSH group was significantly slower than the LEP group. The 10-meter maximum walking speed was significantly faster in the PSH $(0.66 \pm 0.44 \mathrm{~m} / \mathrm{sec})$ and LEP $(1.23 \pm 0.34 \mathrm{~m} / \mathrm{sec})$ groups with AFO than without AFO (Table 2). Furthermore, the percent and actual increase in the 10-meter maximum walking speed upon wearing AFO was significantly greater in the PSH (132\%) than LEP group (111\%) (Table 2).

\section{Distance on the 6-minute walking test}

The distance accomplished in the 6 MWT was significantly shorter in the PSH $(147.3 \pm 108.4 \mathrm{~m})$ and LEP $(311.6 \pm 112.2 \mathrm{~m})$ groups without AFO compared with that of the control group $(473.5 \pm 92.9 \mathrm{~m})$. Furthermore, the distance of 6 MWT walked by patients of the PSH group was significantly shorter than that by the LEP group. Also, the distance completed in the $6 \mathrm{MWT}$ was significantly longer with AFO for both the PSH $(193.7 \pm 118.3 \mathrm{~m})$ and LEP $(352.6 \pm 109.4 \mathrm{~m})$ groups than without AFO (Table 2), but the difference disappeared between control group and LEP group after wearing the AFO by the latter group. The percent and actual increase in the $6 \mathrm{MWT}$ distance with AFO was significantly higher in the PSH group (132\%) than LEP group (113\%) (Table 2).

\section{Berg balance scale}

The total score of BBS was significantly smaller in both the PSH

\begin{tabular}{|c|c|c|c|c|c|c|c|}
\hline & \multirow{2}{*}{$\begin{array}{l}\text { Control without } \\
\text { AFO }\end{array}$} & \multicolumn{3}{|c|}{ PSH } & \multicolumn{3}{|c|}{ LEP } \\
\hline & & without AFO & with AFO & Change (\%) & without AFO & with AFO & Change (\%) \\
\hline 10 MMWS (m/s) & $1.88 \pm 0.45$ & $0.50 \pm 0.41^{\dagger}$ & $0.66 \pm 0.44^{\star}, \dagger$ & 132 & $1.11 \pm 0.31^{\dagger, \#}$ & $1.23 \pm 0.34^{*},+\#$ & $111^{\#}$ \\
\hline $6 \mathrm{MWT}(\mathrm{m})$ & $473.5 \pm 92.9$ & $147.3 \pm 108.4^{\dagger}$ & $193.7 \pm 118.3^{*}, \dagger$ & 132 & $311.6 \pm 112.2^{\dagger, \#}$ & $352.6 \pm 109.4^{*}, \#$ & $113^{\#}$ \\
\hline BBS & $55.7 \pm 0.9$ & $40.7 \pm 8.9^{\dagger}$ & $43.4 \pm 8.4^{\star}+\dagger$ & 107 & $48.6 \pm 10.9^{\dagger}$ & $49.5 \pm 10.9^{*},+\#$ & $102^{\#}$ \\
\hline
\end{tabular}

Data are mean \pm SD. ${ }^{\star} p<0.05$, compared with the AFO and without AFO. ${ }^{\dagger} p<0.05$, compared with the control group. ${ }^{\#} p<0.05$, compared with the PSH and LEP groups. Abbreviations as in Table 1.

Table 2: Results of 10-meter maximum walking speed (10MMWS), 6-minute walk test (6MWT) and total score of the Berg Balance Scale (BBS) with and without AFO 


\begin{tabular}{|c|c|c|c|c|}
\hline & \multicolumn{2}{|c|}{ PSH } & \multicolumn{2}{|c|}{ LEP } \\
\hline & no AFO & AFO & no AFO & AFO \\
\hline Sitting to standing & $3.7 \pm 0.6$ & $3.8 \pm 0.6$ & $3.9 \pm 0.3$ & $3.9 \pm 0.3$ \\
\hline Standing unsupported & $3.8 \pm 0.6$ & $3.8 \pm 0.6$ & $3.7 \pm 1.1$ & $3.7 \pm 1.1$ \\
\hline Sitting with back unsupported but feet supported on floor on a stool & $4.0 \pm 0$ & $4.0 \pm 0$ & $4.0 \pm 0$ & $4.0 \pm 0$ \\
\hline Standing to sitting & $3.8 \pm 0.4$ & $3.9 \pm 0.4$ & $3.9 \pm 0.3$ & $3.9 \pm 0.3$ \\
\hline Transfers & $3.5 \pm 0.7$ & $3.7 \pm 0.6$ & $3.9 \pm 0.3$ & $3.9 \pm 0.3$ \\
\hline Standing unsupported with eyes closed & $3.6 \pm 0.8$ & $3.8 \pm 0.7$ & $3.7 \pm 1.1$ & $3.7 \pm 1.1$ \\
\hline Standing unsupported with feet together & $3.2 \pm 1.2$ & $3.6 \pm 0.9$ & $3.7 \pm 1.1$ & $3.7 \pm 1.1$ \\
\hline Reaching forward with outstretched arm while standing & $2.8 \pm 1.1$ & $3.1 \pm 1.0$ & $3.7 \pm 1.1$ & $3.7 \pm 1.1$ \\
\hline Pick up objective from the floor from a standing position & $3.3 \pm 1.2$ & $3.5 \pm 1.1$ & $3.4 \pm 1.4$ & $3.4 \pm 1.4$ \\
\hline Turning to look behind over left and right shoulders while standing & $3.3 \pm 1.0$ & $3.5 \pm 0.9$ & $3.7 \pm 1.1$ & $3.7 \pm 1.1$ \\
\hline Turn 360 degrees & $2.0 \pm 1.3$ & $2.3 \pm 1.1$ & $3.4 \pm 1.1$ & $3.4 \pm 1.1$ \\
\hline Placing alternate foot on step or stool while standing unsupported & $1.3 \pm 1.4$ & $1.7 \pm 1.4$ & $2.7 \pm 1.5$ & $2.8 \pm 1.5$ \\
\hline Standing unsupported one foot in front & $1.5 \pm 1.5$ & $1.9 \pm 1.5$ & $3.3 \pm 1.1$ & $3.5 \pm 1.2$ \\
\hline Standing on one leg & $0.8 \pm 0.9$ & $0.9 \pm 1.0$ & $1.7 \pm 1.3$ & $2.2 \pm 1.3$ \\
\hline Total score & $40.7 \pm 8.9$ & $43.4 \pm 8.4^{*}$ & $48.6 \pm 10.9$ & $49.5 \pm 10.9^{*}$ \\
\hline
\end{tabular}

Table 3: Results of Berg balance scale with and without AFO.

$(40.7 \pm 8.9)$ and LEP $(48.6 \pm 10.9)$ groups without AFO compared with the control group $(55.6 \pm 0.9)$. However, there was no significant difference between the total scores of the PSH and LEP groups. AFO significantly increased the BBS score in both the PSH (43.4 \pm 8.4$)$ and LEP $(49.5 \pm 10.9)$ groups compared to the respective values without AFO, however, no significant change was noted in each of the 14 components of the test (Tables 2 and 3 ). AFO induced a significantly larger actual and percent increase in BBS in the PSH group (107\%) than LEP group (102\%) (Table 2).

\section{Discussion}

The beneficial effects of AFO on functional ambulation and functional balance have already been described in individuals with PSH [14]. In clinical practice, many therapists and physicians are aware of the beneficial effects of AFO in patients with peripheral nerve palsy.

However, the present study is the first to describe the beneficial effects of AFO on functional ambulation and functional balance in patients with peripheral nerve palsy. Patients with PSH and LEP showed increased 10-meter maximum walking speed, improvement of 6 MWT distance, and better BBS in tests conducted while wearing the AFO.

AFO is generally used to provide mediolateral stability of the ankle in the stance phase, facilitate gait in the swing phase, and provide ankle support. For example, lesser knee flexion and lesser dorsiflexion are observed in the affected limb of PSH individuals with hemiparesis during the swing phase compared with AFO; these changes require circumduction to achieve toe clearance [23]. Most reports on the clinical use of AFO described their beneficial effects on increasing walking speed, improving toe clearance, and increasing walking speed to normalize heelstrike duration through the use of an optimally adjusted plantarflexion stop [23]. While drop foot is a common finding in patients with LEP, we also consider that LEP patients show lesser dorsiflexion upon walking without AFO, like PSH patients. Gait symmetry and energy efficiency are related factors, therefore, patients with reduced physical activity exhibit less efficient gait patterns, compared with healthy subjects [24]. Hesse, et al. [25] showed that the use of AFO improved gait symmetry in patients with equinovarus deformity. The AFO compensated for the mediolateral instability of the ankle joint, prevented foot drop and decreased the degree of hip-hiking during the swing phase, which resulted in reduction of the minimum amount of energy required to cover the distance. Thus, improvement of functional walking capacity brought about by the use of AFO is due to ankle joint stabilization as well as lower energy consumption required for ambulation [26]. The results of the $6 \mathrm{MWT}$ and 10-m maximum walking speed tests in our study were similar to those of other studies and confirmed the beneficial effects of AFO in both PSH and LEP.

With regard to the effects of AFO on BBS, previous studies reported improvement of BBS in patients with stroke $[27,28]$. Analysis of the results of BBS in the present study indicated clear improvement in functional balance during AFO use in patients of both the PSH and LEP groups. The ankle joint has special importance in balanced evaluation by BBS, because the ankle is a key joint in the transfer of body weight to the ground and postural stability. Mojika, et al. [29]. investigated the effect of plastic AFO on body sway in $8 \mathrm{PSH}$ patients and reported that the center of foot pressure moved toward the unaffected limb without AFO, whereas the use of AFO shifted the center of foot pressure towards the mid-position and decreased body sway. We believe that the use of AFO stabilizes the ankle joint and reduces mediolateral instability of the subtalar joint, thus increasing functional balance. While it is common for physiotherapists and physicians to use AFO for PSH patients, the use AFO in LEP patients could improve walking distance and functional balance.

One limitation of this study is the small sample size of the LEP group. The small sample size and the strict inclusion and exclusion criteria may have resulted in the selection of healthier cohort with higher mobility compared to the average patients with LEP and those with chronic PSH. Another limitation of this study was the lack of analysis of the effect of body weight on the recorded variables. Previous studies have reported that obese individuals have poor $6 \mathrm{MWT}$ and poor walking speed [30]. While the body weight was different between the LEP and PSH groups, we did not evaluate the potential effect of body weight on the tested variables. Further large-scale study of patients with various symptoms is needed to confirm the findings of the present study.

\section{Conclusion}

The results of the present study demonstrated the benefits of AFO in patients with PSH and LEP as it increases walking speed, improves walking ability and functional balance. The results could promote the use of AFO and increase compliance in patients with PSH and LEP. 
Citation: Kinoshita T, Nishimura Y, Nakamura T, Kojima D, Sakurai Y, et al. (2016) Ankle Foot Orthosis Improves Functional Ambulation and Balance in Patients with Peripheral Nerve Palsy. J Spine 5: 337. doi: 10.4172/2165-7939.1000337

\section{Acknowledgment}

The authors thank Hiroyuki Tsuboi, Yoshio Yamamoto, Takashi Moriki, Yasuhisa Fujita, Hiroyasu Uenishi, and Ms Mami Yamashiro. We also thank Dr Faiq $\mathrm{G}$ Issa for the careful reading and editing of the manuscript.

\section{Conflict of interest}

None of the authors report a Conflict of interest.

\section{References}

1. Ministry of Health, Labour and Welfare (2016) The general conditions of the patients survey. Accessed on Oct 182016.

2. Ministry of Health, Labour and Welfare (2016) The annual estimation of vita statistics. Accessed on Oct $18^{\text {th }} 2016$.

3. Esquenazi A, Hirai B (1991) Assessment of gait and orthotic prescription. Phys Med Rehab Clin North Am 2: 473-485.

4. Jørgensen HS, Nakayama H, Raaschou HO, Olsen TS (1995) Recovery of walking function in stroke patients: the Copenhagen Study. Arch Phys Med Rehab 76: 27-32.

5. Chan L, Koepsell TD, Deyo RA, Esselman PC, Haselkorn JK, et al. (1997) The effect of Medicare's payment system for rehabilitation hospitals on length of stay, charges and payments. N Engl J Med 337: 978-985.

6. Jorgensen JR, Bech-Pedersen DT, Zeeman P, Sorensen J, Andersen LL, et al. (2010) Effect of intensive outpatient physical training on gait performance and cardiovascular health in people with hemiparesis after stroke. Phys Ther 90: 527-537.

7. Quach L, Galica AM, Jones RN, Procter-Gray E, Manor B, et al. (2011) The nonlinear relationship between gait speed and falls: The maintenance of balance, independent living, intellect, and zest in the elderly of Boston study. J Am Geriatr Soc 59: 1069-1073.

8. Studenski S, Perera S, Patel K, Rosano C, Faulkner K, et al. (2011) Gait speed and survival in older adults. J Am Med Assoc 305: 50-58.

9. International Organization for Standardization, ISO 8549-1: 1989 Prosthetics and Orthotics - Vocabulary. General terms for external limb prostheses and orthoses. International Organization for Standardization: Geneva, Switzerland.

10. Leung J, Mosely A (2003) Impact of ankle-foot orthoses on gait and leg muscle activity in adults with hemiplegia. Physiotherapy 89: 39-55.

11. Jutai J, Coulson S, Teasell R, Bayley M, Garland J, et al. (2007) Mobility assistive device utilization in a prospective study of patients with first-eve stroke. Arch Phys Med Rehabil 88: 1268-1275.

12. Davidson I, Waters K (2000) Physiotherapists working with stroke patients: a national survey. Physiotherapy 86: 69-80.

13. Lennon S, Baxter D, Ashburn A (2001) Physiotherapy based on the Bobath concept in stroke rehabilitation: a survey within the UK. Disabil Rehabil 23 : 254-262.

14. Tyson SF, Kent RM (2013) Effects of an ankle-foot orthosis on balance and walking after stroke: a systematic review and pooled meta-analysis. Arch Phys Med Rehabil 94: 1377-1385.

15. Lehmann JF, Condon SM, De Lateur BJ, Price R (1986) Gait abnormalities in peroneal nerve paralysis and their corrections by orthoses: a biomechanical study. Arch Phys Med Rehabil 67: 380-386.

16. De Bruijn IL, Geertzen JH, Dijkstra PU (2007) Functional outcome afte peroneal nerve injury. Int J Rehabil Res 30: 333-337.

17. De Haan R, Limburg M, Bossuyt P, Van der Meulen J, Aaronson N (1995) The clinical meaning of Rankin 'handicap' grades after stroke. Stroke 26: 2027-2030.

18. Iwata M, Kondo I, Sato Y, Satoh K, Soma M, et al. (2003) An ankle-foot orthosis with inhibitor bar: effect on hemiplegic gait. Arch Phys Med Rehabil 84: 924 927.

19. Gordon HG, Michael JS, Penelope JT, Fallen EL, Pugsley SO, et al. (1985) The 6-minute walk: a new measure of exercise capacity in patients with chronic heart failure. Can Med Assoc J 132: 919-923.

20. Berg K, Wood-Dauphinee S, Williams JI (1995) The Balance Scale: reliability assessment with elderly residents and patients with an acute stroke. Scand J Rehabil Med 27: 27-36.

21. Goldstein LB, Bartels C, Davis JN (1989) Interrater reliability of the NIH stroke scale. Arch Neurol 46: 660-662.

22. Ashworth B (1964) Preliminary trial of carisoprodol in multiple sclerosis. Practitioner 192: 540-542.

23. Lehmann JF, Condon SM, Price R, De Lateur BJ (1987) Gait abnormalities in hemiplegia: their correction by ankle-foot orthoses. Arch Phys Med Rehabil 68 763-771.

24. Waters RL, Mulroy S (1999) The energy expenditure of normal and pathologic gait. Gait Posture 9: 207-231.

25. Hesse S, Werner C, Matthias K, Stephen K, Berteanu M (1999) Non-velocityrelated effects of a rigid double-stopped ankle-foot orthosis on gait and lower limb muscle activity of hemiparetic subjects with an equinovarus deformity Stroke 30: 1855-1861.

26. Corcoran PJ, Jebsen RH, Brengelmann GL, Simons BC (1970) Effects of plastic and metal leg braces on speed and energy cost of hemiparetic ambulation. Arch Phys Med Rehabil 51: 69-77.

27. Simons DC, Van Asseldonk EH, Van der Kooij H, Geurts AC, Buurke JH (2009) Ankle-foot orthoses in stroke: effects on functional balance, weight-bearing asymmetry and the contribution of each lower limb to balance control. Clin Biomech 24: 769-775.

28. Wang R, Yen L, Lee C, Lin P, Wang M, et al. (2005) Effects of an ankle-foo orthosis on balance performance in patients with hemiparesis of different durations. Clin Rehabil 19: 37-44.

29. Mojica JA, Nakamura R, Kobayashi T, Handa T, Morohashi I, et al. (1988) Effect of ankle-foot orthosis (AFO) on body sway and walking capacity of hemiparetic stroke patients. Tohoku J Exp Med 156: 395-401.

30. Brach JS, Van Swearingen JM, FitzGerald SJ, Storti KL, Kriska AM (2004) The relationship among physical activity, obesity, and physical function in community-dwelling older women. Prev Med 39: 74-80. 